Not All COPD Patients Benefit from Prophylactic Noninvasive Ventilation After Scheduled

\title{
Extubation: An Exploratory Study
}

This article was published in the following Dove Press journal:

International Journal of Chronic Obstructive Pulmonary Disease

\author{
Yan Gong ${ }^{1,2}$ \\ Xiaoli Han' \\ Jun Duan (1D) \\ Shicong Huang' \\ 'Department of Respiratory and Critical \\ Care Medicine, The First Affiliated \\ Hospital of Chongqing Medical University, \\ Chongqing 400016, People's Republic of \\ China; ${ }^{2}$ Department of Teaching Affairs, \\ The First Affiliated Hospital of Chongqing \\ Medical University, Chongqing 400016, \\ People's Republic of China
}

Correspondence: Jun Duan

Department of Respiratory and Critical Care Medicine, The First Affiliated

Hospital of Chongqing Medical University, Youyi Road I, Yuzhong District,

Chongqing 400016, People's Republic of China

Tel $+86-23-89012680$

$\mathrm{Fax}+86-23-89012690$

Email duanjun4I2589@I63.com
Background: Prophylactic noninvasive ventilation (NIV) after scheduled extubation can benefit patients with chronic respiratory disorders, among which chronic obstructive pulmonary disease (COPD) is a significant example. However, it is not known whether all COPD patients benefit from prophylactic NIV.

Methods: We performed a post hoc analysis of prospectively collected data. COPD patients who successfully completed a spontaneous breathing trial were enrolled. In the prophylactic NIV group, NIV was applied immediately after extubation. In the usual care group, conventional oxygen therapy was used. Patients were followed up to 90 days post-extubation.

Results: Among patients with $\mathrm{PaCO}_{2}>45 \mathrm{mmHg}, 128$ and 40 received prophylactic NIV and usual care, respectively. Prophylactic NIV led to lower rates of re-intubation (4\% vs $30 \%$ at $72 \mathrm{~h}$ and $11 \%$ vs $35 \%$ at 7 days, both $\mathrm{p}<0.01)$ and hospital mortality $(18 \%$ vs $40 \%, \mathrm{p}<0.01)$ than usual care. The proportion of 90-day mortality was also lower in the prophylactic NIV group (log rank test, $\mathrm{p}=0.04$ ). Among patients with $\mathrm{PaCO}_{2} \leq 45 \mathrm{mmHg}, 32$ and 21 received prophylactic NIV and usual care, respectively. In this cohort however, prophylactic NIV neither reduced reintubation ( $6 \%$ vs $5 \%$ at $72 \mathrm{~h}, \mathrm{p}>0.99$, and $9 \%$ vs $14 \%$ at 7 days, $\mathrm{p}=0.67)$ nor hospital mortality $(19 \%$ vs $24 \%, p=0.74)$. The proportion of 90 -day mortality did not differ between the two groups (log rank test, $\mathrm{p}=0.79$ ).

Conclusion: This exploratory study shows that prophylactic NIV benefits COPD patients with $\mathrm{PaCO}_{2}>45 \mathrm{mmHg}$, but it may not benefit those with $\mathrm{PaCO}_{2} \leq 45 \mathrm{mmHg}$. Further study with a larger sample size is required to confirm this.

Keywords: noninvasive ventilation, ventilator weaning, re-intubation

\section{Introduction}

Chronic obstructive pulmonary disease (COPD) is a worldwide public health challenge, due to its high prevalence and related disability and mortality. ${ }^{1}$ It is the fourth leading cause of death worldwide and the third in China. ${ }^{2,3}$ Among COPD patients who receive invasive mechanical ventilation, hospital mortality reaches $30 \%{ }^{4}$ Investigation of how to reduce mortality in critically ill COPD patients is needed.

Noninvasive ventilation (NIV) is widely used to liberate patients from invasive mechanical ventilation. NIV is performed immediately following endotracheal tube removal (prophylactic use) in patients who successfully complete a spontaneous breathing trial (SBT) to avoid extubation failure. Prophylactic NIV reduces extubation failure in high-risk patients but not in the overall population. ${ }^{5-9}$ Therefore, guidelines recommend that NIV be used preventively in high-risk patients. ${ }^{10,11}$ COPD is a risk 
factor for extubation failure. ${ }^{12,13}$ It has been demonstrated that prophylactic NIV benefits patients who exhibit this risk factor. ${ }^{6,7}$ However, it remains unclear whether all COPD patients benefit from prophylactic NIV. For this reason, we identified COPD patients who could benefit from prophylactic NIV.

\section{Methods}

From 2011 to 2018 , we developed a database in an intensive care unit (ICU) of a teaching hospital and prospectively collected data for patients who were ready for extubation after successful SBT. In this study, we enrolled patients diagnosed with COPD. Data on some patients taken from a previous study were analyzed as well. ${ }^{14}$ However, patients with tracheotomies were excluded. The study protocol was approved by the local ethics committee (the First Affiliated Hospital of Chongqing Medical University). Due to the observational nature of this study, informed consent was waived. We declare that all the data which can identify the personal information has been hidden. And all the interventions are performed as our hospital protocol, which is according with the Declaration of Helsinki.

Patients admitted to our ICU were managed following hospital protocols. COPD was diagnosed based on the criteria published by Chinese Medical Association. ${ }^{15}$ Bronchodilators such as salbutamol and budesonide were used to reduce airway resistance for patients who received invasive mechanical ventilation. Expectorants such as acetylcysteine were used to diminish airway secretions. Sedation and analgesia were used to promote patient-ventilator interaction. At the beginning of ventilation, the assist/control mode was used. After respiratory failure was reversed, pressure support ventilation (PSV) was used to facilitate ventilator weaning.

We screened the patients every morning to identify those who could be liberated from invasive mechanical ventilation. A SBT was performed if the following criteria were met: reversal of the underlying cause of acute respiratory failure, positive end-expiratory pressure $(\mathrm{PEEP}) \leq 5 \mathrm{cmH}_{2} \mathrm{O}, \mathrm{FiO}_{2} \leq$ $50 \%, \mathrm{PaO}_{2} / \mathrm{FiO}_{2} \geq 150$, temperature $\leq 38^{\circ} \mathrm{C}$, heart rate $\leq 120$ beats/min, breathing frequency $\leq 30$ cycles/min, and hemodynamic stability. ${ }^{14}$ PSV was used to perform a $120 \mathrm{~min}$ SBT. Support pressure was set at 6 and $8 \mathrm{cmH}_{2} \mathrm{O}$ for inner diameters of the endotracheal tube of $\geq 7.5$ and $<7.5 \mathrm{~mm}$, respectively. ${ }^{16,17}$ SBT failure was defined as the presence one of the following criteria: heart rate $\geq 140$ or $\leq 50$ beats $/ \mathrm{min}$, breathing frequency $\geq 35$ cycles/min, rapid shallow breathing index $\left(\mathrm{f} / \mathrm{V}_{\mathrm{T}}\right)>105$, peripheral oxygen saturation $\left(\mathrm{SpO}_{2}\right)<$
$90 \%$ at $\mathrm{FiO}_{2} \geq 50 \%$, systolic blood pressure $\geq 180$ or $\leq 90$ $\mathrm{mmHg}$; diminishing consciousness or diaphoresis; and clinical signs indicating respiratory muscle fatigue, labored breathing, or both. The endotracheal tube was removed if there were no signs of SBT failure.

Following their attending physicians' orders, after extubation, patients received immediate use of NIV or conventional oxygen therapy. Based on existing evidence, physicians were more likely to order prophylactic NIV in patients at risk for extubation failure. ${ }^{6-9}$ In our department, we used BiPAP Vision and V60 (Philips Respironics, Monroeville, PA, USA) to perform NIV. The S/T mode was selected. At the beginning of NIV, the expiratory positive airway pressure was set at $4 \mathrm{cmH}_{2} \mathrm{O}$ and gradually increased to counterbalance the intrinsic PEEP. The initial inspiratory pressure was set at $8 \mathrm{cmH}_{2} \mathrm{O}$ and gradually increased to reach a tidal volume of around $8 \mathrm{~mL} / \mathrm{kg}$ or to the maximum tolerated level for each patient. The $\mathrm{FiO}_{2}$ was set to maintain $\mathrm{SpO}_{2}$ around 95\%. After $24 \mathrm{~h}$, liberation from NIV was considered, following hospital protocol. ${ }^{18}$ In patients who received conventional oxygen therapy, a nasal cannula or mask was used. Oxygen flow was adjusted to maintain $\mathrm{SpO}_{2}$ around 95\%.

Reintubation was determined based on the protocol of our hospital (one major criterion or at least two minor criteria). ${ }^{14}$ The major criteria were loss of consciousness, heart rate $<50$ beats/min with loss of alertness, respiratory arrest, development of conditions necessitating intubation to protect the airway (coma or seizure disorders) or copious tracheal secretions requiring management, and hemodynamic instability without response to fluids and vasoactive drugs. The minor criteria were hypoxemia with $\mathrm{PaO}_{2}<60 \mathrm{mmHg}$ at $\mathrm{FiO}_{2}>0.5$ or supplemental oxygen flow $>10 \mathrm{~L} / \mathrm{min}$, acidosis with $\mathrm{pH}$ $<7.30$, breathing frequency $>35$ cycles/min, persistent tachycardia, and persistent activation of accessory respiratory muscles.

At extubation, we recorded the age, gender, acute physiology and chronic health evaluation II score, heart rate, breathing frequency, blood pressure, Glasgow coma scale, hemoglobin, and duration of ventilation. We recorded reintubation at $72 \mathrm{~h}$ and 7 days post-extubation. We also recorded ICU stay, hospital stay, ICU mortality, and hospital mortality. Patients were followed up to 90 days or death, whichever came first.

$\mathrm{PaCO}_{2}$ was measured at the end of SBT. A previous study reported that prophylactic NIV benefited patients with $\mathrm{PaCO}_{2}$ greater than $45 \mathrm{mmHg}$ when measured at 
extubation. $^{7}$ We used this cutoff value to divide the patients into two cohorts.

\section{Statistical Analysis}

Normally distributed continuous variables are reported as mean and standard deviation. Non-normally distributed continuous variables are reported as medians and interquartile ranges. The differences between the groups were analyzed using the independent-sample $T$-test or the Mann-Whitney $U$-test when appropriate. Categorical variables are reported as numbers and percentages, and differences between groups were analyzed using the chi-square and/or Fisher's exact test when appropriate. Kaplan-Meier analysis was used to indicate survival probability. A p value less than 0.05 was taken to indicate statistical significance.

\section{Results}

We enrolled 168 patients with $\mathrm{PaCO}_{2}>45 \mathrm{mmHg}$. Of these, 128 cases received prophylactic NIV, and 40 cases received conventional oxygen therapy. Another 53 patients were enrolled with $\mathrm{PaCO}_{2} \leq 45 \mathrm{mmHg}$. Of these, 32 cases received prophylactic NIV, and 21 cases received conventional oxygen therapy. The demographics collected at extubation are summarized in Table 1.

In the cohort with $\mathrm{PaCO}_{2} \leq 45 \mathrm{mmHg}$, the NIV group had a longer duration of invasive mechanical ventilation before extubation than the control group (with medians [interquartile ranges] of 5 [3-9] vs 4 [2-6] days, $\mathrm{p}=0.04)$. In the cohort with $\mathrm{PaCO}_{2}>45 \mathrm{mmHg}$, the NIV group had lower hemoglobin than the control group $(11.7 \pm 2.3$ vs 12.8 $\pm 2.1 \mathrm{~g} / \mathrm{dL}, \mathrm{p}=0.01)$. There were no differences between the NIV and control groups in either cohort for other variables.

In the cohort with $\mathrm{PaCO}_{2} \leq 45 \mathrm{mmHg}$, there were no differences in the re-intubation rate, hospital mortality, 90-day mortality, ICU stay, or hospital stay between the NIV and control groups (Table 2). The crude and adjusted odds ratios (ORs) for re-intubation at 7 days were 0.62 (95\% confidence interval [CI], 0.11-3.41, $\mathrm{p}=0.58)$ and 0.35 (95\% CI, $0.04-2.79, \mathrm{p}=0.32$ ). In the cohort with $\mathrm{PaCO}_{2}>45 \mathrm{mmHg}$, the NIV group had a lower re-intubation rate and hospital mortality. The crude and adjusted OR of re-intubation at 7 days were $0.23(95 \% \mathrm{CI}, 0.10-0.54, \mathrm{p}<0.01)$ and 0.17 (95\% CI, 0.07-0.46, p < 0.01).

There was no difference in cumulative survival between the NIV and control groups in the $\mathrm{PaCO}_{2} \leq 45 \mathrm{mmHg}$ cohort

Table I Demographic Data Collected at Extubation

\begin{tabular}{|c|c|c|c|c|c|c|}
\hline & \multicolumn{2}{|c|}{$\mathrm{PaCO}_{2} \leq 45 \mathrm{mmHg}$} & \multirow[t]{2}{*}{$P$} & \multicolumn{2}{|c|}{$\mathrm{PaCO}_{2}>45 \mathrm{mmHg}$} & \multirow[t]{2}{*}{$P$} \\
\hline & NIV $N=32$ & Control $\mathbf{N}=21$ & & $N I V N=128$ & Control $N=40$ & \\
\hline \multicolumn{7}{|l|}{ Underlying disease } \\
\hline Hypotension & $15(50 \%)$ & $9(43 \%)$ & $>0.99$ & 44 (34\%) & $13(33 \%)$ & $>0.99$ \\
\hline Diabetes mellitus & $9(28 \%)$ & $6(29 \%)$ & $>0.99$ & $26(20 \%)$ & $6(15 \%)$ & 0.65 \\
\hline Chronic heart disease & $9(28 \%)$ & $4(19 \%)$ & 0.53 & $35(27 \%)$ & $15(38 \%)$ & 0.24 \\
\hline Chronic kidney disease & I (3\%) & $2(10 \%)$ & 0.56 & $6(5 \%)$ & $0(0 \%)$ & 0.34 \\
\hline Chronic liver disease & - & - & - & $9(7 \%)$ & I (3\%) & 0.45 \\
\hline Age, years & $76 \pm 11$ & $7 I \pm 12$ & 0.09 & $72 \pm 10$ & $73 \pm 8$ & 0.46 \\
\hline Male/female & $22 / 10$ & $15 / 6$ & $>0.99$ & $109 / 19$ & $30 / 10$ & 0.15 \\
\hline APACHE II score & $13 \pm 3$ & $12 \pm 3$ & 0.31 & $12 \pm 3$ & $12 \pm 2$ & 0.48 \\
\hline Heart rate, beats/min & $95 \pm 18$ & $91 \pm 18$ & 0.50 & $100 \pm 14$ & $97 \pm 15$ & 0.30 \\
\hline Systolic blood pressure, $\mathrm{mmHg}$ & $132 \pm 16$ & $128 \pm 17$ & 0.37 & $136 \pm 23$ & $13 \mid \pm 19$ & 0.22 \\
\hline Diastolic blood pressure, $\mathrm{mmHg}$ & $69 \pm 12$ & $75 \pm 10$ & 0.37 & $72 \pm 12$ & $75 \pm 10$ & 0.23 \\
\hline GCS & $|4.8 \pm 1|$. & $14.5 \pm 1.6$ & 0.55 & $14.9 \pm 0.3$ & $14.9 \pm 0.5$ & 0.82 \\
\hline Hemoglobin, g/dL & $10.6 \pm 2.1$ & $10.9 \pm 2.0$ & 0.67 & $11.7 \pm 2.3$ & $12.8 \pm 2.1$ & $0.01 *$ \\
\hline Duration of ventilation, $d$ & $5(3-9)$ & $4(2-6)$ & $0.04 *$ & $6(4-8)$ & $5(3-7)$ & 0.26 \\
\hline Breathing frequency, cycles/min & $22 \pm 5$ & $21 \pm 4$ & 0.54 & $2 I \pm 5$ & $22 \pm 5$ & 0.76 \\
\hline Rapid shallow breathing index & $54 \pm 23$ & $51 \pm 26$ & 0.64 & $53 \pm 25$ & $59 \pm 24$ & 0.19 \\
\hline $\mathrm{pH}$ & $7.45 \pm 0.04$ & $7.45 \pm 0.04$ & 0.79 & $7.40 \pm 0.05$ & $7.4 I \pm 0.05$ & 0.33 \\
\hline $\mathrm{PaO}_{2}, \mathrm{mmHg}$ & $93 \pm 22$ & $94 \pm 27$ & 0.86 & $85 \pm 21$ & $91 \pm 21$ & 0.10 \\
\hline $\mathrm{PaCO}_{2}, \mathrm{mmHg}$ & $40 \pm 3$ & $38 \pm 5$ & 0.16 & $60 \pm 10$ & $57 \pm 8$ & 0.07 \\
\hline $\mathrm{PaO}_{2} / \mathrm{FiO}_{2}, \mathrm{mmHg}$ & $240 \pm 63$ & $250 \pm 84$ & 0.63 & $210 \pm 56$ & $222 \pm 55$ & 0.15 \\
\hline
\end{tabular}

Notes: Duration of ventilation is reported as medians and interquartile ranges. ${ }^{*} p<0.05$.

Abbreviations: GCS, Glasgow coma scale; APACHE II, acute physiology and chronic health evaluation II; Min, minimum; Max, maximum. 
Table 2 Outcomes

\begin{tabular}{|c|c|c|c|c|c|c|}
\hline & \multicolumn{2}{|c|}{$\mathrm{PaCO}_{2} \leq 45 \mathrm{mmHg}$} & \multirow[t]{2}{*}{$\mathbf{P}$} & \multicolumn{2}{|c|}{$\mathrm{PaCO}_{2}>45 \mathrm{mmHg}$} & \multirow[t]{2}{*}{$\boldsymbol{P}$} \\
\hline & NIV $\mathbf{N}=32$ & Control $\mathbf{N}=\mathbf{2} I$ & & NIV $N=\mid 28$ & Control $\mathbf{N}=40$ & \\
\hline Re-intubation at $72 \mathrm{~h}$ & $2(6 \%)$ & $\mathrm{I}(5 \%)$ & $>0.99$ & $5(4 \%)$ & $12(30 \%)$ & $<0.01 *$ \\
\hline Re-intubation at 7 days & $3(9 \%)$ & $3(14 \%)$ & 0.67 & $14(11 \%)$ & $14(35 \%)$ & $<0.01 *$ \\
\hline Hospital mortality & $6(19 \%)$ & $5(24 \%)$ & 0.74 & $23(18 \%)$ & $16(40 \%)$ & $<0.01 *$ \\
\hline 90-day mortality & II (34\%) & $8(38 \%)$ & $>0.99$ & 31 (24.2\%) & $16(40.0 \%)$ & 0.07 \\
\hline LOS in ICU, days & $12(8-17)$ & $7(5-14)$ & 0.10 & $13(9-120)$ & $12(8-19)$ & 0.54 \\
\hline LOS in hospital, days & $19(11-24)$ & $17(10-37)$ & 0.90 & $19(13-27)$ & $19(14-27)$ & 0.93 \\
\hline LOS in ICU after extubation, days & $5(3-8)$ & $3(2-7)$ & 0.28 & $7(4-I I)$ & $7(5-11)$ & 0.87 \\
\hline LOS in hospital after extubation, days & $9(5-16)$ & $8(5-14)$ & 0.88 & $12(8-19)$ & II (7-16) & 0.64 \\
\hline
\end{tabular}

Notes: LOS in ICU, LOS in hospital, LOS in ICU after extubation, and LOS in hospital after extubation are reported as medians and interquartile ranges. ${ }^{p} p<0.05$. Abbreviations: NIV, noninvasive ventilation; LOS, length of stay; ICU, intensive care unit.

( $\log$ rank test, $\mathrm{p}=0.79$ ) (Figure 1 ). However, in the cohort with $\mathrm{PaCO}_{2}>45 \mathrm{mmHg}$, it was much lower in the NIV group than in the control group (log rank test, $\mathrm{p}=0.04)$ (Figure 2).

\section{Discussion}

Our study systematically explored the effects of prophylactic NIV in COPD patients. In the cohort with $\mathrm{PaCO}_{2} \leq 45$ $\mathrm{mmHg}$, prophylactic NIV did not reduce re-intubation or hospital mortality. In the cohort with $\mathrm{PaCO}_{2}>45 \mathrm{mmHg}$, prophylactic NIV significantly reduced re-intubation and hospital mortality.

The physiological effects of NIV in COPD patients counterbalance the intrinsic PEEP, which decreases the work of breathing. ${ }^{19}$ Therefore, NIV is widely used in the process of

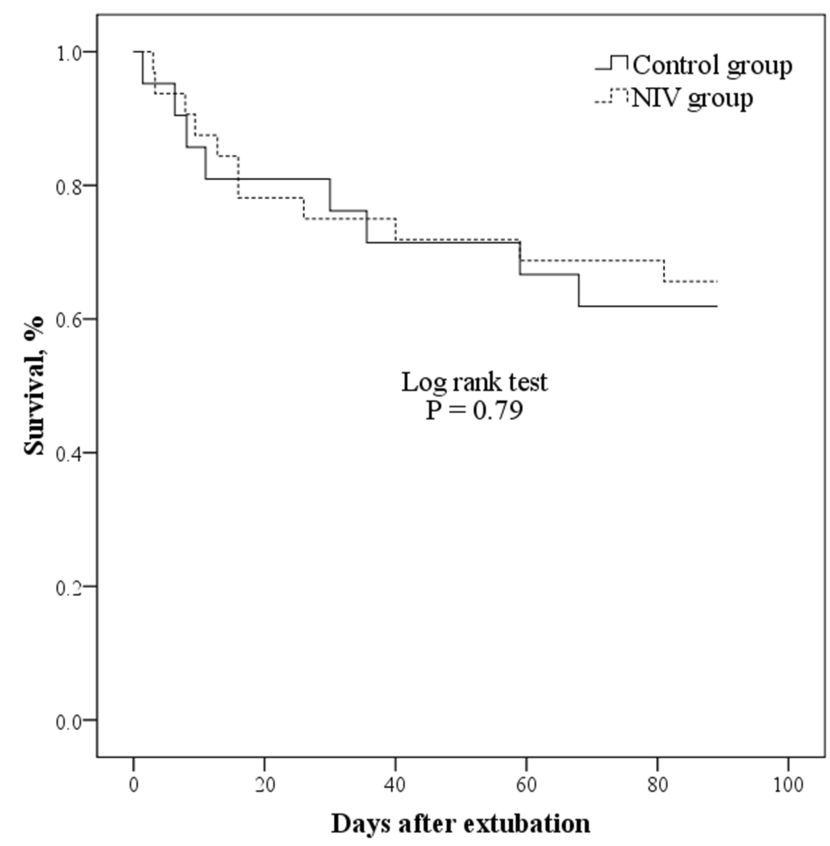

Figure I Survival in patients with $\mathrm{PaCO}_{2} \leq 45 \mathrm{mmHg}$. ventilator weaning. ${ }^{20} \mathrm{~A}$ recent systematic review and metaanalysis showed that NIV as a weaning strategy reduced reintubation in COPD population (relative risk $=0.33$ ). ${ }^{21}$ However, in our study, we challenged the assumption that all COPD patients benefit from prophylactic NIV. We found that prophylactic NIV benefited COPD patients with $\mathrm{PaCO}_{2}$ $>45 \mathrm{mmHg}$ but not in those with $\mathrm{PaCO}_{2}<45 \mathrm{mmHg}$. This is a new insight into the effects of NIV on ventilator weaning.

To the best of our knowledge, only one study has explored the effects of prophylactic NIV in COPD patients. ${ }^{22}$ However, its sample was limited to 20 patients in each group. This small sample size failed to show any benefits from NIV. A further three studies have explored the effects of prophylactic NIV in patients with chronic

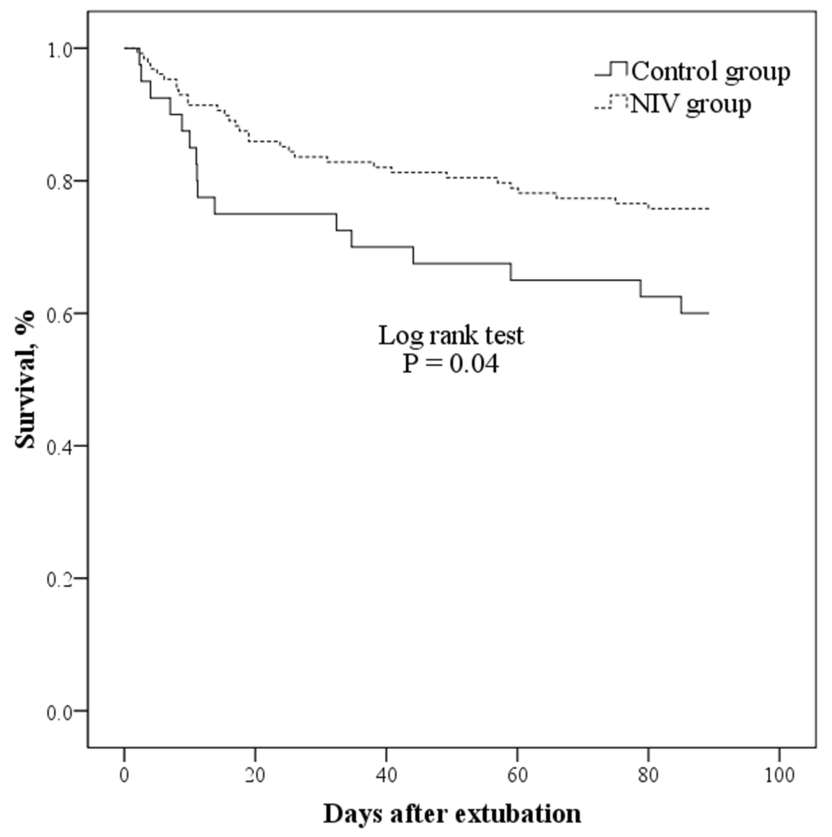

Figure 2 Survival in patients with $\mathrm{PaCO}_{2}>45 \mathrm{mmHg}$. 
respiratory disorders. ${ }^{7,8,23}$ These studies showed that patients with chronic respiratory disorders benefit from prophylactic NIV. However, COPD was only one of the chronic respiratory disorders reviewed. Those studies also failed to unequivocally demonstrate whether COPD patients could benefit from prophylactic NIV. Therefore, the use of prophylactic NIV in COPD patients has remained controversial. Because our study enrolled 221 COPD patients, it enabled us to conduct a subgroup analysis based on the level of $\mathrm{PaCO}_{2}$ measured at extubation, which constitutes a major strength of this study. Furthermore, we found that prophylactic NIV benefited COPD patients with hypercapnia but not nonhypercapnic patients. This can provide a reference for decision makers as they consider how to use NIV in COPD patients after a scheduled extubation.

Among the low-risk patients who received conventional oxygen therapy after a planned extubation, the rate of reintubation at $72 \mathrm{~h}$ post-extubation was $12.2 \% .{ }^{24}$ Among patients with $\mathrm{PaCO}_{2} \leq 45 \mathrm{mmHg}$, the re-intubation rates at $72 \mathrm{~h}$ post-extubation were $6 \%$ and $5 \%$ in the NIV and control groups, respectively. This indicates that these patients had a low risk of re-intubation. A previous study demonstrated that prophylactic NIV did not reduce reintubation or ICU mortality in patients with a low risk of reintubation. ${ }^{5}$ Therefore, prophylactic NIV may not benefit COPD patients with $\mathrm{PaCO}_{2} \leq 45 \mathrm{mmHg}$. However, a recent study reported that high-risk patients with $\mathrm{PaCO}_{2} \leq 45$ mmHg reduced re-intubation. ${ }^{25}$ In this study, only $18 \%$ of patients were diagnosed with COPD in the cohort with $\mathrm{PaCO}_{2} \leq 45 \mathrm{mmHg}$. It means that these patients had other risk factors for re-intubation. Therefore, COPD patients with $\mathrm{PaCO}_{2} \leq 45 \mathrm{mmHg}$ may benefit from prophylactic NIV if they feature other risk factors for re-intubation.

A previous study classified the reasons for re-intubation as airway and non-airway failure. ${ }^{26}$ Airway failure was the inability to breathe without a tracheal tube due to airway obstruction resulting from aspiration or excessive respiratory secretions. Non-airway failure might lead to an inability to breathe without mechanical ventilation due to congestive heart failure, lung disease, or hypoventilation. In the COPD population, $\mathrm{PaCO}_{2}$ is negatively correlated with tidal volume and pulmonary function. ${ }^{27}$ Patients with higher $\mathrm{PaCO}_{2}$ may have lower tidal volumes and worse pulmonary function. ${ }^{27}$ Therefore, the proportion of non-airway failure may be higher in patients with $\mathrm{PaCO}_{2}>45 \mathrm{mmHg}$ than in those with $\mathrm{PaCO}_{2} \leq 45 \mathrm{~mm} \mathrm{Hg}$. NIV can improve outcomes among patients at risk for non-airway failure because it can improve alveolar ventilation and reduce the work of breathing. ${ }^{28}$ However, it is difficult to manage risk factors for non-airway failure. This may be a reason for difficult reintubation rates among patients with $\mathrm{PaCO}_{2} \leq 45$ and $>45$ $\mathrm{mmHg}$ when they have received NIV care.

Our study had several limitations. As it was an observational study, the use of prophylactic NIV was decided by the attending physicians. Patients at high risk for extubation failure were more likely to receive prophylactic NIV than patients with low risk. Hypercapnic patients were more likely to receive prophylactic NIV. This resulted in selection bias. In addition, we only enrolled 53 COPD patients with $\mathrm{PaCO}_{2} \leq 45 \mathrm{mmHg}$. This might have limited statistical power and made it inadequate to conclude that prophylactic NIV did not benefit COPD patients with $\mathrm{PaCO}_{2} \leq 45 \mathrm{mmHg}$. Further studies with larger sample sizes are required to confirm this result.

\section{Conclusions}

This exploratory study shows that prophylactic NIV benefits COPD patients with $\mathrm{PaCO}_{2}>45 \mathrm{mmHg}$, but it may not do so in those with $\mathrm{PaCO}_{2} \leq 45 \mathrm{mmHg}$. Further studies with larger sample sizes are required to draw more robust conclusions on this issue.

\section{Author Contributions}

Jun Duan conceived the study and drafted the manuscript. Yan Gong, Xiaoli Han, Jun Duan and Shicong Huang contributed to the study design, patient recruitment and data collection. Yan Gong contributed to patient follow-up visit. All authors contributed to data analysis, revising the article, gave final approval of the version to be published, and agree to be accountable for all aspects of the work.

\section{Funding}

This study was funded by Chongqing Health Commission Projects (2017MSXM019).

\section{Disclosure}

Dr Jun Duan report grants from Chongqing Health Commission Projects, during the conduct of the study. The authors report no other conflicts of interest in this work.

\section{References}

1. Vos T, Allen C, Arora M. Global, regional, and national incidence, prevalence, and years lived with disability for 310 diseases and injuries, 1990-2015: a systematic analysis for the Global Burden of Disease Study 2015. Lancet. 2016;388:1545-1602. doi:10.1016/ S0140-6736(16)31678-6 
2. Rabe KF, Hurd S, Anzueto A, et al. Global strategy for the diagnosis, management, and prevention of chronic obstructive pulmonary disease: GOLD executive summary. Am J Respir Crit Care Med. 2007;176:532-555. doi:10.1164/rccm.200703-456SO

3. Zhou M, Wang H, Zhu J, et al. Cause-specific mortality for 240 causes in China during 1990-2013: a systematic subnational analysis for the Global Burden of Disease Study 2013. Lancet. 2016;387:251-272. doi:10.1016/S0140-6736(15)00551-6

4. Gadre SK, Duggal A, Mireles-Cabodevila E, et al. Acute respiratory failure requiring mechanical ventilation in severe chronic obstructive pulmonary disease (COPD). Medicine (Baltimore). 2018;97:e0487. doi:10.1097/MD.0000000000010487

5. Su CL, Chiang LL, Yang SH, et al. Preventive use of noninvasive ventilation after extubation: a prospective, multicenter randomized controlled trial. Respir Care. 2012;57:204-210. doi:10.4187/respcare.01141

6. Thille AW, Boissier F, Ben-Ghezala H, et al. Easily identified at-risk patients for extubation failure may benefit from noninvasive ventilation: a prospective before-after study. Crit Care. 2016;20:48. doi:10.1186/s13054-016-1228-2

7. Ferrer M, Sellares J, Valencia M, et al. Non-invasive ventilation after extubation in hypercapnic patients with chronic respiratory disorders: randomised controlled trial. Lancet. 2009;374:1082-1088. doi:10.1016/S0140-6736(09)61038-2

8. Ferrer M, Valencia M, Nicolas JM, et al. Early noninvasive ventilation averts extubation failure in patients at risk: a randomized trial. Am J Respir Crit Care Med. 2006;173:164-170. doi:10.1164/ rccm.200505-7180C

9. Nava S, Gregoretti C, Fanfulla F, et al. Noninvasive ventilation to prevent respiratory failure after extubation in high-risk patients. Crit Care Med. 2005;33:2465-2470. doi:10.1097/01.CCM.0000186416.44752.72

10. Davidson AC, Banham S, Elliott M, et al. BTS/ICS guideline for the ventilatory management of acute hypercapnic respiratory failure in adults. Thorax. 2016;71(Suppl 2):ii1-ii35. doi:10.1136/thoraxjnl-2015-208209

11. Schmidt GA, Girard TD, Kress JP, et al. Official executive summary of an American Thoracic Society/American College of chest physicians clinical practice guideline: liberation from mechanical ventilation in critically Ill adults. Am J Respir Crit Care Med. 2017;195:115-119. doi:10.1164/rccm.201610-2076ST

12. Thille AW, Harrois A, Schortgen F, et al. Outcomes of extubation failure in medical intensive care unit patients. Crit Care Med. 2011;39:2612-2618. doi:10.1097/CCM.0b013e3182282a5a

13. Chu CC, Liu CJ, Yen SM, et al. Factors associated with re-intubation within 14 days after ventilator liberation. Respir Care. 2017;62:1557-1564. doi:10.4187/respcare.05649

14. Duan J, Han X, Huang S, et al. Noninvasive ventilation for avoidance of reintubation in patients with various cough strength. Crit Care. 2016;20:316. doi:10.1186/s13054-016-1493-0

15. Chen W, Qing-yuan Z. Guideline for mechanical ventilation in patients with acute exacerbation of chronic obstructive pulmonary disease (2007). Zhongguo Wei Zhong Bing Ji Jiu Yi Xue. 2007;19:513-518.
16. Boles JM, Bion J, Connors A, et al. Weaning from mechanical ventilation. Eur Respir J. 2007;29:1033-1056. doi:10.1183/09031936.00010206

17. Fiastro JF, Habib MP, Quan SF. Pressure support compensation for inspiratory work due to endotracheal tubes and demand continuous positive airway pressure. Chest. 1988;93:499-505. doi:10.1378/ chest.93.3.499

18. Duan J, Tang X, Huang S, et al. Protocol-directed versus physician-directed weaning from noninvasive ventilation: the impact in chronic obstructive pulmonary disease patients. J Trauma Acute Care Surg. 2012;72:1271-1275. doi:10.1097/TA.0b013e318249a0d5

19. O'Donoghue FJ, Catcheside PG, Jordan AS, et al. Effect of CPAP on intrinsic PEEP, inspiratory effort, and lung volume in severe stable COPD. Thorax. 2002;57:533-539. doi:10.1136/thorax.57.6.533

20. Esteban A, Frutos-Vivar F, Muriel A, et al. Evolution of mortality over time in patients receiving mechanical ventilation. Am J Respir Crit Care Med. 2013;188:220-230. doi:10.1164/rccm.201212-2169OC

21. Bajaj A, Rathor P, Sehgal V, et al. Efficacy of noninvasive ventilation after planned extubation: a systematic review and meta-analysis of randomized controlled trials. Heart Lung. 2015;44:150-157. doi:10.1016/j.hrtlng.2014.12.002

22. Khilnani GC, Galle AD, Hadda V, et al. Non-invasive ventilation after extubation in patients with chronic obstructive airways disease: a randomised controlled trial. Anaesth Intensive Care. 2011;39:217-223. doi:10.1177/0310057X1103900210

23. Vargas F, Clavel M, Sanchez-Verlan P, et al. Intermittent noninvasive ventilation after extubation in patients with chronic respiratory disorders: a multicenter randomized controlled trial (VHYPER). Intensive Care Med. 2017;43:1626-1636. doi:10.1007/s00134-017-4785-1

24. Hernandez G, Vaquero C, Gonzalez P, et al. Effect of postextubation high-flow nasal cannula vs conventional oxygen therapy on reintubation in low-risk patients: a randomized clinical trial. JAMA. 2016;315:1354-1361. doi:10.1001/jama.2016.2711

25. Thille AW, Muller G, Gacouin A, et al. Effect of postextubation high-flow nasal oxygen with noninvasive ventilation vs high-flow nasal oxygen alone on reintubation among patients at high risk of extubation failure: a randomized clinical trial. JAMA. 2019;322:1465-1475. doi:10.1001/ jama.2019.14901

26. Jaber S, Quintard H, Cinotti R, et al. Risk factors and outcomes for airway failure versus non-airway failure in the intensive care unit: a multicenter observational study of 1514 extubation procedures. Crit Care. 2018;22:236. doi:10.1186/s13054-018-2150-6

27. Gorini M, Misuri G, Corrado A, et al. Breathing pattern and carbon dioxide retention in severe chronic obstructive pulmonary disease. Thorax. 1996;51:677-683. doi:10.1136/thx.51.7.677

28. Appendini L, Patessio A, Zanaboni S, et al. Physiologic effects of positive end-expiratory pressure and mask pressure support during exacerbations of chronic obstructive pulmonary disease. Am J Respir Crit Care Med. 1994;149:1069-1076. doi:10.1164/ajrccm.149.5.8173743

\section{Publish your work in this journal}

The International Journal of COPD is an international, peer-reviewed journal of therapeutics and pharmacology focusing on concise rapid reporting of clinical studies and reviews in COPD. Special focus is given to the pathophysiological processes underlying the disease, intervention programs, patient focused education, and self management protocols. This journal is indexed on PubMed Central, MedLine and CAS. The manuscript management system is completely online and includes a very quick and fair peer-review system, which is all easy to use. Visit http://www.dovepress.com/testimonials.php to read real quotes from published authors. 